\title{
Alterações funcionais como consequências de traumatismo orbitário: revisão da literatura
}

Functional changes as orbitary traumatism consequences: literature review

Cambios funcionales como consecuencia del trauma orbitario: revisión de la literatura

\section{Ernest Cavalcante POUCHAIN ${ }^{1}$}

Vanessa Anastácio PIMENTEL ${ }^{2}$

Roque Soares MARTINS NETO

Francisco Willyego de Holanda MACIEL ${ }^{4}$

Kelvin Saldanha LOPES ${ }^{4}$

Bruno da Silva GASPAR ${ }^{5}$

Rafael Linard AVELAR ${ }^{\mathbf{6}}$

${ }^{1}$ Cirurgião Buco-Maxilo-Facial, Mestre em Clínica Odontológica - UFC, Fortaleza - CE

${ }^{2}$ Graduada Pelo Centro Universitário Católica de Quixadá UNICATÓLICA, Quixadá, CE - Brasil

${ }^{3}$ Cirurgião Buco-Maxilo-Facial, Mestrando em Ciências Odontolológicas, Unichritus, Fortaleza - CE

${ }^{4}$ Aluno de graduação em Odontologia do Centro Universitário Católica de Quixadá UNICATÓLICA, Quixadá, CE

${ }^{5}$ Aluno de Graduação em Odontologia da Unichristus, Fortaleza - CE

\section{Resumo}

${ }^{6}$ Doutor em Cirurgia e Traumatologia Buco-Maxilo-Facial pela PUC-RS, Docente do Curso de Odontologia da Unichristus, Fortaleza-CE

Os traumas de face ocorrem por forças externas lesionando o corpo, podendo eles ser locais, gerais ou concomitantes. A etiologia do traumatismo orbitário é diversificada como: quedas, queimaduras e agressões, sendo considerada uma das principais causas de morte no mundo de acordo com Organização Mundial da Saúde (OMS). As fraturas faciais podem se dividir em fraturas dos terço superior, terço médio e terço inferior. As fraturas do tipo Blow-out mantêm as margens orbitais integra, envolvendo apenas parede orbital inferior e ou média. Diplopia e enoftalmia são complicações bem características de traumas orbitais. O objetivo do artigo é identificar as principais lesões ocasionadas por fraturas orbitárias e apontar o diagnóstico e tratamento das lesões. Trata-se de uma revisão de literatura do tipo descritiva, com os dados colhidos nas bases de dados PubMed, SciElo, Lilacs, Google Acadêmico, selecionando artigos entre o ano de 2008 - 2018, de língua inglesa, portuguesa e espanhola, disponíveis para download nas bases de dados citadas. Os exames de imagem como a Tomografia Computadorizada é de suma importância para o diagnóstico devido seu detalhamento. Alguns sinais clínicos são: diplopia, enoftalmia, hipoftalmia ou mobilidade muscular ocular prejudicada. O tratamento ainda varia muito entre os cirurgiões.

Descritores: Diplopia; Fraturas Ósseas; Órbita; Traumatismo do Nervo Abducente; Nervo Óptico.

\section{Abstract}

Face traumas occur by external forces injuring the body, and may be local, general or concomitant. The etiology of orbital trauma is diversified as: falls, burns and aggressions, being considered one of the main causes of death in the world according to the World Health Organization (WHO). Fractures may be divided into fractures of the upper third, middle third and lower third. The fractures of the Blow-out type maintains the orbital margins integrating, involving only the lower and orbital wall. Diplopia and enophthalmia are very characteristic complications of orbital trauma. Fractures may be divided into fractures of the upper third, middle third and lower third. The fractures of the Blow-out type maintains the orbital margins integrating, involving only the lower and orbital wall. Diplopia and enophthalmia are very characteristic complications of orbital trauma. The aim of the article is to identify the main lesions caused by orbital fractures and to point out the diagnosis and treatment of the lesions. This is a literature review of the descriptive type, with the data collected in the databases PubMed, SciElo, Lilacs, Google Academic, selecting articles between the year 2008 - 2018, English, Portuguese and Spanish, available for download in the cited databases. Imaging tests such as Computed Tomography are of paramount importance for diagnosis because of their detail. Some clinical signs are: diplopia, enophthalmia, hypophthalmia or impaired ocular muscle mobility. The treatment still varies greatly among surgeons.

Descriptors: Diplopia; Fractures, Bone; Orbit; Abducens Nerve Injury; Optic Nerve.

\section{Resumen}

El trauma facial ocurre por fuerzas externas que lastiman el cuerpo, que puede ser local, general o concomitante. Según la Organización Mundial de la Salud (OMS), la etiología del trauma orbitario es diversa, como caídas, quemaduras y agresiones, siendo considerada una de las principales causas de muerte en todo el mundo. Las fracturas faciales se pueden dividir en fracturas del tercio superior, el tercio medio y el tercio inferior. Las fracturas por reventón mantienen los márgenes orbitales integrales, involucrando solo las paredes orbitales inferiores y medias. La diplopía y la enoftalmia son complicaciones muy características del trauma orbitario. El objetivo del artículo es identificar las principales lesiones causadas por fracturas orbitales y señalar el diagnóstico y el tratamiento de las lesiones. Esta es una revisión descriptiva de la literatura, con datos recopilados en las bases de datos PubMed, SciElo, Lilacs, Google Scholar, seleccionando artículos de 2008 a 2018 , disponibles en inglés, portugués y español, disponibles para descargar. en las bases de datos citadas. Las imágenes como la tomografía computarizada son de suma importancia para el diagnóstico debido a sus detalles. Algunos signos clínicos son: diplopía, enoftalmia, hipoftalmia o movilidad muscular ocular deteriorada. El tratamiento aún varía ampliamente entre los cirujanos.

Descriptores: Diplopía; Fracturas Óseas; Órbita; Traumatismo del Nervio Abducente; Nervio Óptico.

\section{INTRODUÇÃO}

Traumas são descritos pelo deslocamento de força de um meio externo para o corpo que sofrerá as lesões, contusões e fraturas. Assim os ferimentos são lesões que afetam os tecidos moles das vítimas ${ }^{1}$. A associação de alterações funcionais e anatômicas é considerada trauma facial, pode ser classificado como geral local ou combinado. Sua etiologia é diversificada, sendo causadas por quedas, queimaduras e agressões. Independente da cultura, condição financeira ou idade, qualquer pessoa pode ser acometida por estes fatos, já que são doenças mais frequentes em saúde pública ${ }^{2}$. Conforme informações da Organização Mundial da Saúde (OMS), uma das causas primordiais de mortes no mundo são por algum tipo de traumatismo. Diariamente, 16.000 indivíduos falecem por consequência de alguma espécie de trauma ${ }^{3}$.

Em 1844, Mackenzie iniciou o detalhamento das fraturas do assoalho orbital ${ }^{4}$. Anatomicamente as rupturas faciais são classificadas em fraturas do terço superior (osso frontal até a borda supra orbital); terço médio (zigomático, maxila, nasais e orbital); terço inferior (mandíbula e dentoalveolar) ${ }^{5}$.

As rupturas orbitais são uma parte do 
traumatismo facial. A fratura blow-out (BOF), é um tipo de fratura orbital, na qual corresponde a uma fratura com envolvimento apenas da parede orbital inferior e/ou média mantendo a margem orbital integra. A fratura é uma consequência do trauma orbital. Os resultados da BOF incluem enoftalmia, diplopia, paralisia do nervo infra-orbitário, assimetria facial e obstrução do ducto nasolacrimal ${ }^{6}$.

As rupturas do zigoma e dos ossos circundantes são algo comum na prática clínica. Diversas nomenclaturas foram utilizadas para determinar esses tipos de trauma, entretanto a Fratura Zigomático Orbital (ZMO) é a mais adequada, já que elas envolvem os ossos da cavidade orbital. É a fratura mais comum vista pelos clínicos em sua prática. As fraturas de ZMO podem causar disfunções funcionais e estéticas. As alterações funcionais podem ocasionar diplopia, disfunção do nervo infraorbitário e trismo ${ }^{7}$.

O traumatismo facial é considerado o mais perceptível, devido suas sequelas emocionais e funcionais e a probabilidade de deformações permanentes $^{3}$. As fraturas mais comuns na face média são as fraturas orbitais e as reconstruções das paredes orbitais são bastante complexas já que a sua anatomia é tridimensional $(3 \mathrm{D})^{8}$.

As fraturas ósseas na órbita são comuns em pacientes com trauma na face e/ou crânio. Os traumas nos tecidos concomitantes as lesões dentro da órbita por diversas vezes resultam em complicações bem caracterizadas como diplopia, enoftalmia. A ocorrência de diplopia posteriormente a fraturas orbitais varia de $15 \%$ a $86 \% \%^{9}$.

$\mathrm{O}$ dano e a união ao tecido circundante dos músculos, posição anormal dos músculos, deficiência óssea orbital e degeneração do tecido adiposo orbital são também causas significativas de diplopia, que estão envolvidos em um modo complexo e resultam em movimentos incomuns do globo ocular ${ }^{10}$.

A diplopia severa frequentemente gera amplas incapacidades físicas quando a fratura orbital não recebe tratamento adequado. Diagnosticando e tratando imediatamente, essa fratura orbital reduz o risco de diplopia pós-traumática. O tratamento de rupturas orbitais é abundantemente relatado na literatura, com abordagens filosóficas que variam entre tratamento conservador e cirurgia imediata ${ }^{9}$.

As fraturas ZMO possuem diversos níveis de gravidade. Algumas sofreram um deslocamento pequeno, em outras fraturas o seu deslocamento orbital foi maior. $\mathrm{O}$ aprisionamento da musculatura extra-ocular ocorrerá em uma pequena porção dos casos. Em alguns o paciente pode sofrer significativas perturbações orbitais internas. Dessa maneira para cada fratura ZMO existe um tratamento individualizado, porém esse tratamento varia amplamente entre os cirurgiões ${ }^{7}$.

O planejamento virtual é uma alternativa inicial do tratamento, suas ferramentas devem ser rápidas, distintas e de simples uso, para facilitar ao cirurgião a análise interna e assim decidir o tratamento especifico. A Tomografia Computadorizada (TC) 3D de alta resolução de dados é necessária para todos os tipos de técnicas cirúrgicas ${ }^{8}$.

O objetivo do estudo é, por meio da revisão da literatura, identificar as principais lesões ocasionadas por fraturas orbitárias e apontar o diagnóstico e tratamento das lesões.

\section{MATERIAL E MÉTODO}

$\mathrm{O}$ presente artigo trata-se uma revisão de literatura do tipo descritiva, com dados colhidos nas bases de dados: PubMed, SciElo, Lilacs, Google Acadêmico. Os artigos selecionados para a pesquisa foram publicados entre os anos de 2008 e 2018. Durante a análise e escolha dos materiais para estudo foram imposto os critérios de inclusão e exclusão no estudo. Foram selecionados 42 artigos e escolhidos 23. Os critérios de inclusão foram artigos na língua inglesa, portuguesa e espanhola, disponíveis para download nas bases de dados citadas, casos clínicos, pesquisas e revisão de literatura. Trabalhos não disponíveis na integra, livros, monografias, tese, língua francesa foram os critérios de exclusão. Os descritores utilizados foram "Diplopia", "Fraturas Ósseas", "Órbita", "Traumatismo do Nervo Abducente" e "Nervo Óptico".

\section{RESULTADOS E DISCUSSÃO}

Diferente do que muitos pensam e falam a cavidade orbitária não tem o seu assoalho plano, pelo contrário a anatomia do assoalho orbitário é bastante complexa devido às faixas do piso que devem se unir de forma quase plana e essa união se difere a cada pessoa $^{11,12}$. Segundo Palmieri e Ghali ${ }^{13}$ a cortical das bordas orbitárias é constituída por uma cortical densa, protegendo o conteúdo orbital e globo de possíveis traumas diretos. Entretanto, Tavares et al. ${ }^{14}$ e Long e Gutta ${ }^{15}$ afirmam que devido a anatomia da cavidade orbitária (terço médio da face), se encontra exposta significativamente aos traumatismos e consequentemente as fraturas de blow-out, fraturas do teto da órbita, fraturas naso-órbito-etmoidais, fraturas da parede medial e lateral da órbita, podendo essas fraturas se tornarem complexas. Porém, Long e $\mathrm{Gutta}^{15}$ justificam sua afirmação dizendo que a estrutura óssea é fraca. He et al. ${ }^{11}$ e Roth et al. ${ }^{12}$ afirmam que a parede medial devido sua espessura está mais propícia as fraturas ${ }^{11,12}$, enquanto Palmieri e Ghali ${ }^{13}$ explicam que o assoalho da orbital está como a parede medial favorável a fraturas.

Palmieri e Ghali ${ }^{13}$ relatam que o volume do globo em média dos adultos é de $30 \mathrm{ml}$ e que por volta dos 5 anos de idade o crescimento orbital está com $85 \%$ completo e finalizado quando a criança 
completa 7 anos de idade podendo atingir até a puberdade. Já nos idosos ocorre uma reabsorção do teto da órbita.

Tavares et al. ${ }^{14}$ apontam que as fraturas orbitárias ocorrem mais rotineiramente em indivíduos masculinos e jovens, tendo como origem agressão, trauma esportivo e acidentes automobilísticos. É importante ressaltar que esse tipo de fratura gera consequências importantes na função e estética nas áreas lesionadas. Roth et al. ${ }^{12}$ concordam com a origem dos traumas de acordo Tavares et al. ${ }^{14}$, mas acrescentam os assaltos como outra alternativa. Palmieri e Ghali ${ }^{13}$ concordam com os dois autores anteriores.

De acordo com Long e Gutta ${ }^{15}$ as deformidades orbitais traumáticas podem variar de uma fratura orbital isolada a uma fratura complexa com o envolvimento total da órbita. Palmieri e Ghali $^{13}$ são mais detalhistas, e especificam em porcentagens que as fraturas orbitárias isoladas representam de $4 \%$ a $16 \%$ de todas as fraturas faciais, mas se as fraturas do complexo zigomático e naso-orbital-etmóidal estejam inclusas a representação irá de $30 \%$ a $55 \%$ de todas as fraturas faciais. He et al. ${ }^{11}$ destacam que os dois tipos principais de fraturas combinadas sustentadas em seu estudo foram: fratura orbitária medial-inferior e fratura orbitária lateral-inferior, concordando com Palmieri e Ghali ${ }^{13}$ que as fraturas mais comuns de acontecer são as do complexo zigomático e a nasoorbital-etmóidal. Wolff et al. ${ }^{16}$ afirmam que as fraturas isoladas do assoalho orbital, parede medial e teto envolvem apenas o esqueleto interno orbital interno, e são constantemente descritas como fraturas blow-out e blow-in.

Segundo Dean et al. ${ }^{17}$ os pacientes podem ter ou desenvolver alguns sinais clínicos: diplopia, enoftalmia, hipoftalmia ou alteração da mobilidade ocular. As complicações oftalmológicas são: diplopia, enoftalmia e em casos raros pode ter a perda de visão, acrescenta também anormalidades de contorno, deformidades nas pálpebras juntamente com os outros sinais citados que podem apresentar-se secundariamente $^{13}$. Para Roth et al. ${ }^{12}$ os sinais clínicos mais comuns encontrados em adulto em fraturas orbitárias são: equimoses periorbitais e hemorragias subconjuntivais. Nos casos de ausência de tais sinais não se descarta uma fratura do assoalho orbital, particularmente em pacientes pediátricos ${ }^{12,14}$. Os principais sinais e sintomas das fraturas orbitárias são dor no local da fratura, crepitação, alteração do sulco órbitopalpebral, equimose, limitação dos movimentos oculares, diplopia, parestesia infraorbitária e da pálpebra superior, hematoma e enoftalmia. Para Wolff et al. ${ }^{16}$ a diplopia e enoftalmia são sintomas de um dano ocular agudo, como também são as sequelas mais frequente nas fraturas blow-out quando são tratadas tardias. Fraturas de órbita envolvendo as paredes mediais, laterais e assoalho da órbita são as principais causas de enoftalmia, visto que nessas áreas é a principal para o suporte e correta projeção anterior do globo ocular $^{11,15}$.

De acodo com Roth et al. ${ }^{12}$ as complicações que devem ser observadas com mais importância são: neuropatia óptica secundária ao impacto do trauma, hemorragia ou edema orbital. O impacto extra-ocular é a segunda complicação mais importante, pois pode não ser facilmente administrada secundariamente mesmo com a cirurgia de estrabismo. Porém as complicações mais comuns são enoftalmia e ectrópio.

O deslocamento completo do globo ocular intacto para o seio maxilar é um fato de incidência muito rara após uma extensa fratura blow-out. Apenas 04 pacientes que sofreram a luxação traumática de um globo ocular intacto no seio maxilar foram relatados ${ }^{18}$.

De acordo com Dasmaceno e Dasmaceno ${ }^{18}$ isquemia das estruturas da órbita (músculo reto inferior, músculo oblíquo inferior e, às vezes, a gordura periorbital) ocorrem muito rápido, e quando acontece essa fratura passa a ser uma urgência terapêutica, necessitando de tratamento cirúrgico em até 48 horas. Para Palmieri e Ghali ${ }^{13}$ vários fatores devem ser considerados para determinar qual o tipo de intervenção cirúrgica em pacientes com fraturas orbitária. Roth et al. ${ }^{12}$ e Long e Gutta ${ }^{15}$ relatam que o tratamento cirúrgico muitas vezes é tratado de forma semiatrasada, em torno de $07-14$ dias, antes que ocorra cicatrização dos tecidos moles e após o alívio do edema pós traumático, pois no período maior de 02 semanas os tecidos moles podem sofrer fibrose, principalmente em pacientes do sexo masculino e jovens e será necessário uma dissecação aguda para redução de tecidos orbitais. O edema deve ser reduzido visto que o edema no intraoperatório pode distorcer a posição do globo e aumentar o risco de congestão orbital pós-operatória e perda de visão. O paciente que receber o tratamento inadequado, como o atraso excessivo na intervenção cirurgica pode estar associado ao aumento das complicações pósoperatórias, tais como: enoftalmia, restrição da mobilidade ocular, distopia ocular ou orbital, e defeitos nos tecidos moles ${ }^{15}$. He et al. ${ }^{11}$ ressalvam que em casos com enoftalmia maior que $2 \mathrm{~mm}$ o seu tratamento será a intervenção cirúrgica. Wolff et al. ${ }^{16}$ defendem outro critério para a escolha do tratamento nos casos em que o paciente apresente os sinais clínicos como diplopia, enoftalmia, distúrbios nos movimentos oculares e defeitos no piso ou paredes da órbita maiores de $10 \mathrm{~mm}$ de diâmetro decorrentes de fraturas indicam a necessidade de tratamento cirúrgico, a redução aberta.

CONCLUSÃO

Conclui-se a partir deste trabalho, que as 
principais lesões decorrentes das fraturas orbitárias são: diplopia, enoftalmia, hipoftalmia ou mobilidade muscular ocular comprometida e em raríssimos casos, a perda da visão do paciente. O diagnóstico pode ser feito no exame clínico, quando observado os sinais e sintomas como, hematomas, equimose conjuntival, parestesia infraorbitária, edemas locais, fragmentação dos segmentos ósseos fraturados, telecanto traumático, ptose palpebral e dor. Os exames de imagem como radiografia e TC auxiliam na identificação das fraturas, porém, a TC é o melhor exame de imagem devido os detalhes que são possíveis de serem observados nesse exame. O tratamento das lesões varia de acordo com o tipo e tamanho das fraturas, como a abordagem do cirurgião, que pode ser a cirurgia imediata ou conservadora.

\section{REFERÊNCIAS}

1. Ramos JC, Almeida MLD, Alencar YCG, de Sousa Filho LF, Figueiredo CHMC, Almeida MSC. Estudo epidemiológico do trauma bucomaxilofacial em um hospital de referência da Paraíba. Rev Col Bras Cir. 2018;45(6):e1978.

2. Affonso PRA, Cavalcanti MA, Groisman S, Gandelman I. Etiologia de trauma e lesões faciais no atendimento pré - hospitalar no Rio de Janeiro. Rev UNINGÁ. 2010;23(1):23-34.

3. Scolari N, Heitz C. Protocolo de tratamento em fraturas orbitárias. RFO UPF. 2012;17(3):365-69.

4. Polligkeit J, Grimm M, Peters JP, Cetindis M, Krimmel M, Reinert S. Assessment of indications and clinical outcome for the endoscopy-assisted combined subciliary/transantral approach in treatment of complex orbital floor fractures. J Craniomaxillofac Surg. 2013;41(8):797-802.

5. Mendonça JCG, Freitas GP, Lopes HB, Nascimento VS. Tratamento de fraturas complexas do terço médio da face: relato de caso. Rev Bras Cir Craniomaxilofac 2011;14(4):221-24.

6. Jung H, Byun JY, Kim HJ, Min JH, Park GM, Kim HY, Kim YK, Cha J, Kim ST. Prognostic CT findings of diplopia after surgical repair of pure orbital blowout fracture. J Craniomaxillofac Surg. 2016;44(9):1479-84.

7. Ellis E 3rd, Perez D. An algorithm for the treatment of isolated zygomatico-orbital fractures. J Oral Maxillofac Surg. 2014;72(10):1975-83.

8. Nilsson J, Nysjö J, Carlsson AP, Thor A. Comparison analysis of orbital shape and volume in unilateral fractured orbits. J Craniomaxillofac Surg. 2018;46(3):381-87.

9. Yu DY, Chen CH, Tsay PK, Leow AM, Pan CH, Chen CT. Surgical Timing and Fracture Type on the Outcome of Diplopia After Orbital Fracture Repair. Ann Plast Surg. 2016;76 Suppl 1:S91-5.

10. Morotomi T, Iuchi T, Hashimoto T, Sueyoshi Y, Nagasao $T$, Isogai $\mathrm{N}$. Image analysis of the inferior rectus muscle in orbital floor fracture using cine mode magnetic resonance imaging. J Craniomaxillofac Surg. 2015;43(10):2066-70.

11. He Y, Zhang Y, An JG. Correlation of types of orbital fracture and occurrence of enophthalmos. J Craniofac Surg. 2012;23(4):1050-53.

12. Roth FS, Koshy JC, Goldberg JS, Soparkar CN. Pearls of orbital trauma management. Semin Plast Surg. 2010;24(4):398-410.

13. Palmieri CF Jr, Ghali GE. Late correction of orbital deformities. Oral Maxillofac Surg Clin North Am. 2012;24(4):649-63.

14. Tavares SSS, Tavares GR, Oka SC, Cavalcante JR, Paiva MAF. Fraturas orbitárias: revisão de literatura e relato de caso. Rev Cir Traumatol Buco-Maxilo-Fac. 2011;11(2):35-42.

15. Long JA, Gutta R. Orbital, periorbital, and ocular reconstruction. Oral Maxillofac Surg Clin North Am. 2013;25(2):151-66.

16. Wolff J, Sándor GK, Pyysalo M, Miettinen A, Koivumäki AV, Kainulainen VT. Late reconstruction of orbital and naso-orbital deformities. Oral Maxillofac Surg Clin North Am. 2013;25(4):683-95.

17. Dean A, Heredero S. Alamillos F.J, García-García B. Aplicación clínica de la planificación virtual y la navegación en el tratamiento de las fracturas del suelo de la órbita. Rev Esp Cir Oral Maxilofac. 2015; 37(4):220-28.

18. Damasceno NAP, Damasceno EF. raumatic orbital fracture with intact ocular globe displacement into the maxillary sinus. Rev bras oftalmol. 2010;69(1):52-4.

\section{CONFLITO DE INTERESSES}

Os autores declaram não haver conflitos de interesse.

\section{AUTOR PARA CORRESPONDÊNCIA}

\section{Rafael Linard Avelar}

R. João Adolfo Gurgel, 133 - Cocó, 60190-180 Fortaleza - CE, Brasil 